Laien senken lässt. Dies war lediglich in drei der Studien ein Thema. In einer von ihnen wurde die Mortalität durch Laienhilfe um $6 \%$ gesenkt, allerdings in einem Kriegsgebiet. Da hier zum großen Teil Schussverletzungen erfasst wurden, kann das Ergebnis nicht auf Unfälle mit stumpfen Traumen übertragen werden. Verlässlicher scheinen die Resultate zweier retrospektiver Autopsiestudien. Hier schätzten die Autoren, dass 4,5\% bzw. 1,8\% der Menschen hätten überleben können, wenn Laien Erste Hilfe geleistet hätten.

\section{Inaktiv bei schweren Verletzungen}

Als Ursachen dafür, dass Menschen an Unfallorten inaktiv bleiben, vermuten die Autoren insbesondere die Schwere der Verletzungen, Angst, die Unfähigkeit, Verletzungen zu erkennen, oder die Erwartung, dass der Krankenwagen sowieso gleich eintreffe.

Zwar ist es nach Ansicht der Autoren wegen der Heterogenität der Studien noch immer schwierig, den Nutzen der Laienhilfe am Unfallort $\mathrm{zu}$ beurteilen, doch scheint sich ein Vorteil für die Unfallopfer abzuzeichnen. Allein dieser rechtfertige es, darüber nachzudenken, wie man ErsteHilfe-Maßnahmen von Laien in Notfallsituationen mit stumpfen Traumen weiter verbessern könne.

(Christine Starostzik)

Tannvik TD et al, A systematic literature review on first aid provided by laypeople to trauma victims, Acta Anaesthesiol Scand 2012 (Online first)

Bloß kein Übereifer

\title{
Bei der Herzmassage kostet Schnelligkeit Tiefe
}

Die zur Reanimation empfohlene Rate an Thoraxkompressionen von $100-120 /$ min sollte nicht zu weit überschritten werden. Je höher die Frequenz ist, desto geringer fällt die Kompressionstiefe aus.

Geschwindigkeit und Tiefe der Herzdruckmassage wurden bei 133 Patienten gemessen, die außerhalb eines Krankenhauses von professionellen Helfern wiederbelebt wurden. Maßgabe für die Reanimationsmaßnahmen war die damals geltende Leitlinie des European Resuscitation Council (ERC) aus dem Jahr 2005. In dieser Leitlinie war noch eine Tiefe von $4-5 \mathrm{~cm}$ bei einer Frequenz von ungefähr 100/min empfohlen. Die aktuelle ERC-Leitlinie von 2010 fordert, das Brustbein „mindestens $5 \mathrm{~cm}$ (aber nicht mehr als $6 \mathrm{~cm}$ )“ in Richtung Wirbelsäule zu drücken, und das mit einer Rate von „mindestens 100/min (aber nicht mehr als $120 / \mathrm{min}) “$.

\section{Nur 30\% leitlinienkonform}

Jeder Studienpatient erhielt durchschnittlich 1605 Thoraxkompressionen. Davon wurden $2 \%$ (möglicherweise wegen Unterbrechungen) mit einer Rate unter $80 / \mathrm{min}$ appliziert, $62 \%$ mit $80-120 / \mathrm{min}$ und $36 \%$ mit über 120/min. Die Eindrücktiefe lag zu $36 \%$ unter $4 \mathrm{~cm}$, zu $45 \%$ bei $4-5 \mathrm{~cm}$ und zu 19\% über $5 \mathrm{~cm}$. Damit wurden nur 30\% der Kompressionen leitlinienkonform durchgeführt.

Kompressionen mit einer Frequenz über 120/min waren bei der Mehrzahl der Patienten (58\%) weniger tief als Kompressionen mit 80-120/min. Bei 30\% der Patienten erreichte dieser Unterschied sogar mindestens $0,5 \mathrm{~cm}$ und wurde damit als klinisch signifikant eingestuft. Die tiefste Kompression ist nach dieser Studie bei einer Frequenz von 86/min zu erwarten. Bei Raten von 100 bzw. 120/min wurden schätzungsweise 4,5 bzw. $4,3 \mathrm{~cm}$ Tiefe erreicht. Eine zu schnelle Herzmassage mit einer Frequenz über 145/min resultierte in einer Eindrücktiefe von nur noch $4 \mathrm{~cm}$.

Die Frequenz bei der Thoraxkompression sollte 145/min nicht überschreiten, um Kompressionen mit ungenügender Tiefe zu vermeiden.

(bs)

Monsieurs KG et al, Resuscitation 2012 (Online first)

\section{Hier steht eine Anzeige.}

